\title{
Effects of chrysotherapy on circulating lymphocyte numbers and subsets
}

\author{
M D SMITH, ${ }^{1}$ A SMITH ${ }^{2}$ M FEHR, ${ }^{1}$ M J AHERN ${ }^{2}$ AND \\ P J ROBERTS-THOMSON ${ }^{1}$
}

From the ${ }^{1}$ Department of Clinical Immunology, Flinders Medical Centre, Bedford Park, South Australia, 5042; and the ${ }^{2}$ Rheumatology Unit, Repatriation Hospital, Daws Road, Daw Park, South Australia 5041

SUMMARY In a prospective 24 week study of 25 patients with rheumatoid arthritis (RA) weekly intramuscular (IM) sodium aurothiomalate resulted in a small but significant reduction in the circulating lymphocyte count. Analysis of absolute levels of pan T cells, T4 helper cells, T8 suppressor cells, T4/8 ratio, B cells, and major histocompatibility complex (MHC) class II positive cells showed reductions in these subsets, though these changes did not reach significance. At entry there was no association between circulating lymphocyte counts and subsets and clinical and laboratory indices which reflected disease activity, and during the study gold responders could not be differentiated from non-responders with regard to changes in lymphocyte counts and subsets. Thus this study suggests that weekly IM gold leads to a modest reduction in circulating lymphocyte numbers which involves most subsets. This effect appears to be independent of the clinical efficacy of this drug.

Key words: rheumatoid arthritis, gold.

The mechanism for the efficacy of chrysotherapy in the suppression of rheumatoid inflammation is poorly understood. A large variety of effects on cellular and humoral immune parameters have been noted, but the relevance and interrelationship of these changes are unclear. A recent report has recorded a fall in circulating lymphocyte numbers in 10 patients with RA in response to gold therapy. ${ }^{1}$ The fall was substantial $(40 \%$ of initial lymphocyte count) with the nadir occurring 10-12 weeks after the start of gold therapy and appeared to involve all lymphocyte subsets studied. ${ }^{2}$ The authors concluded that the suppressive effects of gold therapy on circulating lymphocyte numbers might allow new and novel insights into the mechanism of action of gold salts in RA. In contrast, Ishigami et al using three separate statistical methods failed to find a trend in the absolute lymphocyte count in a retrospective study of 58 patients with RA treated with gold salts over a 12 month period (unpublished data). Because of these conflicting results we have

Accepted for publication 27 June 1987.

Correspondence to $\operatorname{Dr} \mathbf{P} \mathbf{J}$ Roberts-Thomson, Department of Clinical Immunology, Flinders Medical Centre. Bedford Park. South Australia 5042 . enumerated circulating lymphocyte numbers and their subsets at two weekly and two monthly intervals respectively in 25 patients with RA undergoing chrysotherapy. The results show a modest but significant fall in circulating lymphocyte numbers which appeared to involve all subsets studied and occurred in both gold responders and nonresponders.

\section{Patients and methods}

PAT IENTS

Twenty five patients with definite or classical RA completed a 24 week course of weekly IM sodium aurothiomalate (total dose $1130 \mathrm{~g}$ ). The diagnosis of RA was in accord with American Rheumatism Association criteria, and 22 of the patients were seropositive. There were 16 women and nine men with a mean age of 64 years and a mean duration of disease of 9.3 years. All patients continued to receive non-steroidal anti-inflammatory drugs and supplemental analgesics during the study, three continued with small amounts of fixed doses of prednisolone, and two continued with hydroxychloroquine. Two patients received three $1 \mathrm{~g}$ intravenous pulses of methylprednisolone at entry into 
the study. All other medications were unaltered during the study.

\section{CLINICAL ASSESSMENT}

Patients were assessed clinically by the same observer at weeks $0,4,8,16$, and 24 weeks of the study. Their articular index was determined (max possible score 78) together with a visual analogue score for pain (max possible score 30 ) and stiffness (max possible score 30 ), and physician global assessment ( $\max$ possible score 30 ). The sum of these figures gave a clinical score ( $\max$ possible 168). Gold responders were defined as those patients whose final clinical score at week 24 was $\leqslant 50 \%$ of the initial value, whereas non-responders were defined as those showing $\leqslant 20 \%$ change.

\section{LYMPHOCYTE MEASUREMENT}

Circulating lymphocyte counts were determined at two weekly intervals and lymphocyte subset analysis was performed at weeks $0,4,8,16$, and 24 . Total white cell count was measured using the Coulter $\mathrm{S}$ plus 6 analyser and a 100 cell differential performed manually. Lymphocyte subset percentages were determined by flow cytometry (fluorescence activated cell sorter IV) and monoclonal antibodies on peripheral blood mononuclear cell preparations as previously described ${ }^{3}$ with the exception that B cells were enumerated according to the presence of surface membrane immunoglobulin. Monocytes in the peripheral blood mononuclear cell preparation were gated out and multiple analyses using FMC33 (a monoclonal antibody reacting with the monocyte/ macrophage lineage) showed that these cells constituted $<5 \%$ of the analysed lymphocytes.
SEROLOG ICAL STUDIES

Serological studies were performed at weeks $0,4,8$ 16 , and 24. Rheumatoid factor (RF), C reactive protein (CRP), and IgG, IgA, and IgM werg measured by rate nephelometry (Beckmans ICS) whereas circulating immune complexes (CIC) wer measured by the fluid phase $\mathrm{Clq}$ binding method of Zubler et al. $^{4}$

STATISTICAL ANALYSIS

Multianalysis of variance was computed using the SPSS package on the Flinders University primo computer. Pearson's correlations were used to compare variables at entry into this study. All dat were $\log$ transformed before analysis. Significance was accepted if $\mathrm{p}<0 \cdot 05$.

\section{Results}

During the 24 week period of this study a small but significant decline in circulating lymphocyte nunf bers occurred with the lowest counts occurring between weeks 16 and $20(p=0.01$, Table 1$)$. transient smaller drop between week 0 and 6 was also observed. Analysis of lymphocyte subset alsg showed lower numbers of pan T cells, T4 helper, T suppressor, B cells, and MHC class II positive celf during the 24 week period as compared with the initial values, but these reductions did not reach significant levels of $p<0.05$ (Table 1). The T4/T ratio also declined owing to a proportionate greater loss of $\mathrm{T} 4$ cells, but again this did not reach significance (Table 1). At entry into this study the lymphocyte count did not correlate significantly wiţô. either clinical score, CRP, RF, CIC, or immun $\bar{Q}$

Table 1 Clinical, serological, and cellular indices during 24 weeks of chrysotherapy $(n=25)$

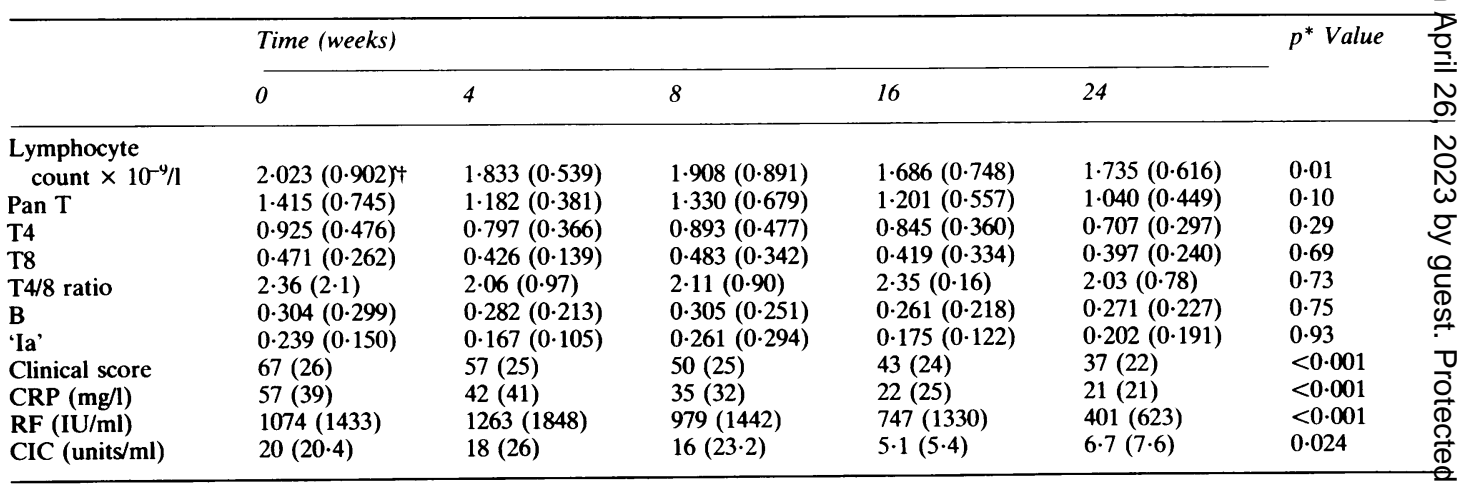

${ }^{*} p=$ probability (analysis of variance with respect to time).

+ Values are mean (SD).

$\mathrm{CRP}=\mathrm{C}$ reactive protein, $\mathrm{RF}=$ rheumatoid factor, $\mathrm{CIC}=$ circulating immune complexes 
Table 2 Multiple analysis of variance of gold responders versus non-responders with respect to time

\begin{tabular}{lllllllll}
\hline & $\begin{array}{l}\text { Clinical } \\
\text { score }\end{array}$ & $\begin{array}{l}\text { Lympho- Pan T } \\
\text { cyte count }\end{array}$ & T4 & T8 & T4/T8 & $\begin{array}{c}\text { B } \\
\text { MHC } \\
\text { positive }\end{array}$ \\
\hline $\begin{array}{l}\text { Responders }(\mathrm{n}=8) v \text { non-responders } \\
(\mathrm{n}=8)\end{array}$ & $<0.001^{*}$ & 0.586 & 0.054 & 0.282 & 0.894 & 0.143 & 0.418 & 0.172 \\
\hline
\end{tabular}

${ }^{*}$ Probability (p) value.

MHC=major histocompatibility complex.

globulins. There was no significant difference during the study between the fall in lymphocyte numbers and subsets and the response to gold therapy, i.e., the declines were observed in both groups to similar degrees (Table 2). When the data were analysed excluding data for the two patients given pulse methylprednisolone in the first week of the study $(n=23)$ the results were the same as when they were included $(n=25)$, and in particular the transient smaller fall in lymphocyte numbers between weeks 0 and 6 was still apparent.

\section{Discussion}

This study has shown that weekly IM gold in RA leads to a small (maximum of $20 \%$ ) reduction of circulating lymphocyte counts which appears to affect cell subsets studied and occurs in both gold responders and non-responders. Thus this study has confirmed the conclusion of Hassan et al, ${ }^{2}$ though the magnitude of the fall seen in our study was smaller.

The mechanism for the reduction of the circulating lymphocyte count is unknown. As it affects most subsets, including the T8 subset which is relatively deficient in RA and appears to be independent of the therapeutic efficacy of gold, it probably has little clinical relevance. As most subsets were affected in a non-selective and time dependent fashion we suggest that sodium aurothiomalate suppresses lymphopoiesis rather than causing redistribution of circulating lymphocytes, though we cannot exclude the possibility that gold is shortening the lymphocyte half life. We also noted in certain individuals that the fall in lymphocyte count was more pronounced while in others it was minimal or not apparent, and this effect did not correlate with clinical outcome.
This suggests that individuals vary in their susceptibility to this gold effect.

It was noted in this study that there was a substantial and significant fall in RF levels (and to a lesser extent IgM, data not shown), while there were no significant changes in circulating surface membrane immunoglobulin positive B lymphocyte numbers. There are a number of possible explanations for this apparent paradox but one of interest is the possibility that gold salts have a selective suppressive effect on the B subpopulation that produces RF (e.g., CD5 positive $\mathrm{B}$ lymphocytes). ${ }^{5}$ This possibility is currently under investigation.

We thank Drs R A Geddes and W R Hill for contributing patients to this study, Mrs M Brown for expert secretarial and typing skills. Mrs J Smith for statistical advice, Mr J Webster for technical assistance with flow cytometry, and May and Baker Australia Pty Ltd for providing financial support. Dr M D Smith is a grateful recipient of an NH \& MRC post-graduate research scholarship.

\section{References}

1 Hanly J G, Bresnihan B. Reduction of peripheral blood lymphocytes in patients receiving gold therapy for rheumatoid arthritis. Ann Rheum Dis 1985; 44: 299-301.

2 Hassan J, Hanly J, Bresnihan B, Feighery C, Whelan C A. The immunological consequences of gold therapy: a prospective study in patients with rheumatoid arthritis. Clin Exp Immunol 1986; 63: 614-20.

3 Bertouch J V, Roberts-Thomson P J, Brooks P M, Bradley J. Lymphocyte subsets and inflammatory indices in synovial fluid and blood of patients with rheumatoid arthritis. $J$ Rheumatol 1984; 11: 754-9.

4 Zubler R H, Nydegger V, Perrin L H, et al. Circulating and intra-articular immune complexes in patients with rheumatoid arthritis. Correlation of $\mathrm{I}^{125} \mathrm{Clq}$ binding activity with clinical and biological features of the disease. J Clin Invest 1976; 57: 1308-19.

5 Lydyard P M, Youinou P Y, Cooke A. CD5-positive B cells in rheumatoid arthritis and chronic lymphocytic leukaemia. Immunol Today 1987; 8: 37-9. 